\title{
Reperméabilisation microchirurgicale après vasectomie. Résultats et facteurs de réussite.
}

\author{
Gert R. DOHLE, Marij SMIT
}

Andrology unit, Department of Urology, Erasmus University Medical Centre, Rotterdam, Pays Bas

\section{RESUME}

La reperméabilisation microchirurgicale post-vasectomie constitue un défi pour le médecin mais le succès du traitement repose sur l'expérience et l'adresse du chirurgien. La fécondité peut être souvent restaurée, épargnant ainsi le recours aux techniques de reproduction artificielles. Les procédures chirurgicales peuvent aussi être combinées avec le recueil de spermatozoïdes et la cryopréservation, permettant une utilisation pour l'injection intracytoplasmique d'un spermatozoïde (ICSI) en cas d'échec de la chirurgie. Nous rapportons les résultats de 217 procédures de vaso-vasostomie, en faisant ressortir plus particulièrement les dernières subtilités techniques ainsi que les indicateurs pronostiques.

Entre 1998 et 2002, nous avons réalisé 217 procédures de vaso-vasostomie dans le cadre d'un centre de référence. Une reperméabilisation a été obtenue dans $76,5 \%$ des cas, une grossesse spontanée est survenue chez $42 \%$ des couples après un suivi d'au moins 1 an. Les principaux facteurs déterminant l'issue de la chirurgie sont l'intervalle écoulé entre la vasectomie et la reperméabilisation ainsi que l'âge de la partenaire : la reperméabilisation a été de $89 \%$ après un intervalle de moins de 5 ans avec la survenue d'une grossesse chez $56 \%$ de ces couples. Après un intervalle de plus de 10 ans, la reperméabilisation diminue à $75 \%$ et le taux de grossesse tombe à $24 \%$. Une dysfonction de l'épididyme, avec une mauvaise mobilité des spermatozoïdes, et une obstruction secondaire de l'épididyme sont fréquentes après un intervalle long. En outre, chez les couples dont la partenaire est âgée de plus de 35 ans, le taux de grossesse n'est que de $21 \%$, indiquant qu'une réserve ovarienne limitée constitue un facteur important pour le résultat final.

Chez les hommes ayant un long intervalle obstructif entre la vasectomie et la reperméabilisation, une obstruction de l'épididyme peut survenir par éclatement du canal épididymaire avec une fuite secondaire du liquide épididymaire et des spermatozoïdes dans l'organe et une fibrose. Une procédure de vaso-épididymostomie est nécessaire pour traiter cette obstruction. Récemment, des subtilités techniques, telle que la technique de l'invagination, ont été introduites pour la procédure de vaso-épididymostomie, donnant des premiers résultats prometteurs. Cette technique simplifiée permet à des micro-chirurgiens moins expérimentés de réaliser cette difficile opération avec succès.

Les résultats de la reperméabilisation post-vasectomie peuvent être augmentés de façon substantielle si le chirurgien est capable de réaliser une vaso-épididymostomie lors d'une obstruction épididymaire secondaire, ce qui arrive chez environ $25 \%$ des hommes qui ont un intervalle de plus de 10 ans.

Mots clés : infécondité masculine, microchirurgie, reperméabilisation post-vasectomie, vaso-épididymostomie

\section{INTRODUCTION}

En Urologie, on a principalement recours à la microchirurgie en cas d'infécondité masculine obstructive ou lors de la réparation d'une varicocèle. Les autres indications sont constituées par la dysfonction érectile vasculaire et les traumatismes vasculaires du testicule ou du pénis. Les obstructions de l'appareil génital mâle représentent 5 à $10 \%$ des causes d'infécondité masculine, et une réparation chirurgicale est possible chez 70 à $80 \%$ de ces hommes [11].

En Europe de l'ouest, environ 10 à $15 \%$ de la population masculine fait confiance à la vasectomie comme méthode contraceptive. Depuis que le nombre de divorces augmen-

\footnotetext{
Correspondance :

Dr G.R. DOHLE, MD, Ph.D - Dept. Urology - Erasmus University Medical Centre Rotterdam, P.O. Box 2040, 3000 CA Rotterdam, The Netherlands

Email g.r.dohle@erasmusmc.nl
} 
te, la demande de reperméabilisation post-vasectomie est croissante : une vaso-vasostomie est couramment faite chez 2 à $6,5 \%$ des hommes vasectomisés [7].

Nous avons analysé les résultats des procédures de reperméabilisation microchirurgicale post-vasectomie en portant notre attention sur le rôle pronostique de l'intervalle entre la vasectomie et la vaso-vasostomie (VS) ainsi que l'âge de la partenaire dans la détermination du résultat final.

\section{MATERIEL ET METHODES}

II s'agit d'une étude rétrospective réalisée sur 217 procédures de reperméabilisation post-vasectomie effectuées entre 1998 et 2002.

En résumé, l'opération était effectuée de la façon suivante : une libération scrotale haute du testicule est réalisée qui permet au chirurgien de faire une anastomose, même si cette dernière doit être effectuée en région inguinale très basse au cas où le site de la vasectomie y serait localisé. Une incision verticale est préférée car elle peut être prolongée à la région inguinale si nécessaire. Le site de la vasectomie précédente est identifié et les deux bouts du canal déférent sont mobilisés pour une anastomose libre de toute tension. On évite attentivement tout stripping du canal puisque cela sera source de problèmes circulatoires et de sténose secondaire du canal déférent. La perméabilité de la partie distale (inguinale) du canal déférent est testée en chassant une faible quantité de solution saline dans la lumière au moyen d'un angiocathèter de calibre 24 . On excise sous microscope tout tissu fibreux qui peut être facilement identifié par des bandes blanches cicatricielles de la couche séromusculaire du canal déférent.

La partie proximale du canal déférent est coupée d'une façon identique, et tout liquide provenant de cette partie testiculaire du canal déférent est examiné au microscope optique à la recherche de spermatozoïdes. L'absence de liquide ou de spermatozoïdes est considérée comme une indication d'inspecter l'épididyme : si l'épididyme est entièrement dilaté, la dissection du canal déférent est poursuivie jusqu'à l'obtention d'un "sperme" de bonne qualité dans une partie plus proximale. Sinon, on réalise une exploration épididymaire avec aspiration microchirurgicale de "sperme" épididymaire (MESA), on recueille des spermatozoïdes mobiles pour une cryopréservation, à visée d'utilisation en
FIV/ICSI si nécessaire, et on effectue une procédure de vaso-épididymostomie (VES) [9].

Une analyse de sperme est réalisée deux fois dans les suites, 3 mois et 6 mois après l'opération. Au moins une année après la procédure chirurgicale, les couples ont été interrogés sur la survenue d'une grossesse spontanée ou l'utilisation de techniques de reproduction artificielle (ART).

\section{RESULTATS}

Nous avons réalisé 217 procédures de reperméabilisation après vasectomie entre 1998 et 2002 . L'âge moyen des hommes était de 46 ans et celui des partenaires de 31 ans. L'intervalle moyen entre la vasectomie et la vaso-vasostomie était de 9,3 ans. Des complications sont survenues chez 24 hommes (11\%), principalement des hématomes et des infections de la cicatrice. II n'y a eu aucune complication majeure. La reperméabilisation fut un succès dans $76,5 \%$ des cas, une grossesse spontanée survint chez $42 \%$ des couples après un suivi d'au moins 1 an. Une azoospermie est apparue chez 46 des 217 hommes (21\%), dans les suites immédiates lors de la première analyse de sperme après chirurgie chez 34 hommes, dans l'année suivante pour 7 cas, et après un délai plus long chez 5 autres.

\section{Rôle de l'intervalle de temps}

Dans cette série de 217 procédures de vaso-vasostomie (VV), 118 hommes avaient un intervalle de temps (entre la vasectomie et la vaso-vasostomie) de plus de 10 ans. La Figure 1 montre les résultats en terme de taux de reperméabilisation et taux de grossesse à des intervalles de temps différents entre la vasectomie et la WS : le taux de reperméabilisation après un intervalle de moins de 5 ans est de $89 \%$, le taux de grossesse étant de $56 \%$ chez ces derniers. Après un intervalle de plus de 10 ans, le taux de reperméabilisation s'abaisse à $75 \%$ et le taux de grossesse spontanée chute à $24 \%$.

Le Tableau 1 rapporte les résultats des analyses de sperme après les 217 procédures de vaso-vasostomie. La mobilité des spermatozoïdes est significativement plus basse quand l'intervalle de temps entre la vasectomie et la WVS est de plus de 10 ans. Les résultats de la numération de sperme, et du taux d'anticorps anti-spermatozoïdes ne sont pas

Tableau 1 : Résultats de la numération de spermatozoïdes, de la mobilité progressive des spermatozoïdes et des anticorps anti-spermatozoïdes (MAR test) en fonction de la durée de l'intervalle obstructif entre la vasectomie et la reperméabilisation.

\begin{tabular}{lccccc}
\hline $\begin{array}{l}\text { Numération de } \\
\text { spermatozoïdes }\end{array}$ & $\begin{array}{c}\mathbf{1 , 0} \text { Mill./ml } \\
(\%)\end{array}$ & $\begin{array}{c}\mathbf{1 , 0 - 2 0} \text { Mill./ml } \\
\mathbf{( \% )}\end{array}$ & $\begin{array}{c}\mathbf{2 0} \text { Mill./ml } \\
\mathbf{( \% )}\end{array}$ & $\begin{array}{c}\text { Mobilité } \\
<\mathbf{1 0 \%}(\%)\end{array}$ & $\begin{array}{c}\text { MAR-test } \\
\text { positif (\%) }\end{array}$ \\
\hline Intervalle obstructif & $8 / 62$ & $34 / 62$ & $20 / 62$ & $9 / 30$ & $29 / 41$ \\
$<10$ ans & $(12,9)$ & $(54,8)$ & $(32,3)$ & $(30,0)$ & $(70,7)$ \\
Intervalle obstructif & $14 / 77$ & $42 / 77$ & $21 / 77$ & $25 / 46$ & $18 / 31$ \\
$>10$ ans & $(18,2)$ & $(54,5)$ & $(27,3)$ & $(54,3)$ & $(58,1)$ \\
Test t de Student & N.S. & N.S. & N.S. & P = 0,025 & N.S. (P=0,49) \\
\hline
\end{tabular}

N.S. : Non significatif. 


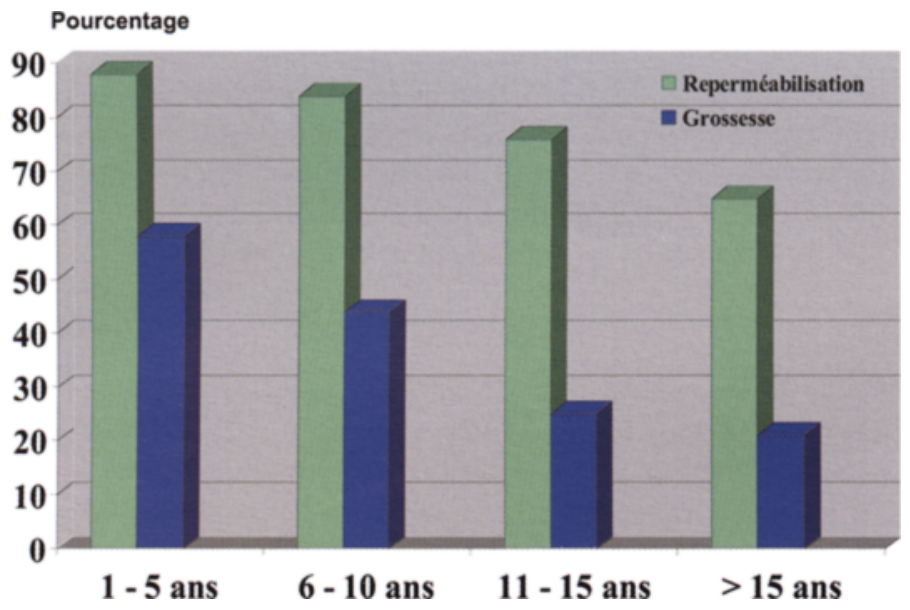

Figure 1 : Taux de reperméabilisation et de grossesse après vaso-vasostomie pour des intervalles de temps différents entre la vasectomie et la chirurgie de reperméabilisation.

significativement différents entre les hommes ayant un intervalle court et ceux ayant un intervalle long.

\section{Rôle de l'âge de la partenaire}

Dans cette série de 217 patients, 30 hommes avaient une partenaire âgée de plus de 35 ans. La moyenne d'âge de ces femmes était de 38,5 ans (extrêmes : $36-43$ ans). Seize de ces femmes avaient déjà eu un enfant dans une précédente relation. L'intervalle moyen entre la vasectomie et la vaso-vasostomie chez ces 30 hommes fut de 12,0 ans. L'analyse de sperme après la vaso-vasostomie montra une oligozoospermie chez $19 / 30(63,3 \%)$ et une normozoospermie chez seulement 4 hommes. Le taux de reperméabilisation fut de $76,6 \%$ et une grossesse spontanée survint chez 6 des 30 couples $(20,0 \%)$.

\section{DISCUSSION}

La réparation microchirurgicale après vasectomie peut constituer une procédure très fructueuse puisque les résultats sont élevés à la fois en terme de reperméabilisation et de grossesses dans le cas de bonnes conditions chirurgicales et après un intervalle de temps court entre la vasectomie et la vaso-vasostomie. L'avantage de la microchirurgie est qu'elle permet au chirurgien de réaliser un alignement délicat et exact entre la partie proximale et la partie distale du canal déférent [9].

Le succès opératoire de la procédure de réversion de la vasectomie dépend de plusieurs facteurs :

- Une manipulation délicate des tissus, avec une préservation attentive des vaisseaux nourriciers du canal déférent.

- Un alignement précis muqueuse à muqueuse.

- Une anastomose étanche sans tension.

- Une exérèse du tissu déférentiel fibreux.
- La présence de spermatozoïdes dans le canal déférent proximal (testiculaire).

- Une anastomose microchirurgicale du canal déférent en 2 plans ou en un plan modifié.

- Des sutures diathermiques bipolaires double-point au fil 9-zéro et 10-zéro non résorbable.

Une sténose tardive de l'anastomose est rapportée chez environ 12 à $18 \%$ des patients dans l'année suivante [18]. Un échec après une première tentative de vaso-vasostomie est habituellement la conséquence d'une constriction de l'anastomose et d'un blocage épididymaire. Une dysfonction épididymaire ou une insuffisance testiculaire ne sont cause d'échec que chez quelques hommes.

\section{Facteurs pronostiques}

Silber a été le premier à décrire une relation inverse entre la durée de l'intervalle obstructif et les taux de reperméabilisation et de grossesse. Le taux de grossesse après VVS diminue progressivement avec la durée de l'intervalle obstructif [24]. Même si chez les hommes ayant un intervalle de plus de 15 ans le taux de reperméabilisation peut être aussi élevé que 60 à $70 \%$, le taux de grossesse n'est que de 20 à $30 \%$, principalement en raison d'une perte de la fonction épididymaire $[2,14]$.

L'âge de la partenaire est un puissant facteur prédictif dans le traitement des couples inféconds [21]. Le déclin de la fonction ovarienne chez la femme "âgée" entraîne une diminution du taux de grossesse, que ce soit naturellement ou avec utilisation de techniques de reproduction artificielle. A partir des résultats obtenus avec ces techniques chez les femmes plus âgées, on estime que le potentiel de fécondité d'une femme âgée de 35 ans n'est que $50 \%$ de celui d'une femme âgée de 25 ans ; à l'âge de 38 ans, il est réduit à $25 \%$, et au-delà de 40 ans il est de moins de $5 \%$. Kolletis et al. ont rapporté une diminution majeure du taux d'accouchement par cycle d'ICSI chez les femmes âgées de plus de 36 ans : le taux chute à $34 \%$ chez celles âgées de 30 à 36 ans, à $13 \%$ chez les femmes âgées de 37 à 39 ans, et à $4 \%$ seulement chez celles de 40 ans et plus [15].

Il a été suggéré qu'aux hommes demandeurs d'un traitement pour une infertilité par vasectomie et ayant des partenaires âgées, il devait être proposé un prélèvement de spermatozoïdes et une ICSI plutôt qu'une vaso-vasostomie. Récemment, Deck et Berger ont comparé les résultats de la reperméabilisation chirurgicale chez des hommes avec une partenaire ayant une ovulation et âgée de plus de 37 ans aux résultats de la récupération de spermatozoïdes et ICSI pour des femmes de la même tranche d'âge [6]. Le taux de naissance après un suivi d'au moins 1 an est de $17 \%$ après reperméabilisation chirurgicale et de $8 \%$ par cycle dans le groupe ICSI. Malgré un taux de grossesse bas dans les deux groupes, les auteurs ont conclu que la reperméabilisation chirurgicale était le traitement de choix pour les hommes dont la partenaire est âgée de plus de 37 ans. Le prélèvement chirurgical de sperme associé à l'ICSI (MESAICSI) n'augmente pas le résultat final de ces cou- 
ples. D'un point de vue coût-efficacité, la vaso-vasostomie est meilleure que la MESAICSI.

Chez les hommes avec un long intervalle de temps, on observe une diminution à la fois de la numération de spermatozoïdes et de la mobilité progressive (Tableau 1). Le faible nombre de spermatozoïdes mobiles peut être la conséquence d'une insuffisance épididymaire, des substances oxygénées réactives (ROS) et des anticorps anti-spermatozoïdes [22, 19].

Le rôle de l'épididyme est le stockage et la maturation des spermatozoïdes, et la traversée de cet organe est essentiel à une fécondation normale. Une insuffisance épididymaire peut être observée après un long intervalle d'obstruction avec dilatation du canal épididymaire. Les ROS, générées par les leucocytes, affectent les spermatozoïdes immatures qui sont fortement sensibles à l'oxydation puisque les enzymes nécessaires à la neutralisation des ROS sont absents des cellules germinales. La leucocytospermie peut résulter d'une obstruction épididymaire et d'une infection à bas bruit de l'appareil génital [22]. Une obstruction de l'épididyme doit être suspectée pendant la chirurgie si aucun liquide ne peut être obtenu au niveau du canal déférent proximal ou bien si le liquide ne contient aucun spermatozoïde.

Des anticorps anti-spermatozoïdes sont retrouvés dans 20 à $50 \%$ des éjaculâts après l'opération [19]. Ces anticorps peuvent entraîner une agglutination des spermatozoïdes et une faible mobilité. Les anticorps IgA de la surface des spermatozoïdes se lient aux anti-lgA du mucus cervical, empêchant toute progression des spermatozoïdes en direction de la cavité utérine. Le lavage des spermatozoïdes et l'insémination intra-utérine sont indiqués chez les couples avec des taux importants d'lgG et d'IgA et une faible mobilité des spermatozoïdes.

Une oligozoospermie sévère avec faible numération et mobilité réduite des spermatozoïdes est habituellement la conséquence d'une constriction de l'anastomose en raison d'un alignement microchirurgical imprécis de la lumière du canal, d'une mauvaise vascularisation du canal déférent ou d'une fuite de liquide séminal entraînant granulome et fibrose [4]. Ces hommes doivent être traités par une nouvelle procédure de reperméabilisation chirurgicale plutôt que par les techniques de reproduction artificielles, puisque à la fois les taux de reperméabilisation et de grossesse sont d'un niveau acceptable après une seconde procédure [8].

La répétition d'une procédure chirurgicale de reperméabilisation est indiquée chez les hommes ayant une reperméabilisation initiale suivie d'une sténose tardive de l'anastomose induisant la survenue d'une oligozoospermie sévère ou d'une azoospermie. Une obstruction partielle avec oligozoospermie et azoospermie survenant après de bons résultats à l'examen de sperme en post-opératoire se produit dans 12 à $18 \%$ des cas [18].

Une seconde procédure chirurgicale de reperméabilisation donne un taux de reperméabilisation de 50 à $80 \%$ et un taux de grossesse spontanée de 20 à 50\%[12]. C'est pourquoi une nouvelle tentative de reperméabilisation chirurgi- cale avec prélèvement de spermatozoïdes et congélation est la première option thérapeutique à proposer à ces couples.

\section{Techniques de reproduction artificielles ou chirurgie ?}

Un recueil chirurgical de spermatozoïdes associé à une ICSI a été préconisé comme le traitement de choix de l'infertilité masculine obstructive, indépendamment de l'étiologie. Toutefois, comparés à ceux de la FIV/ICSI, les résultats du traitement microchirurgical de l'azoospermie obstructive sont largement en faveur du traitement chirurgical [10]. Des taux de grossesse de 44 à $65 \%$ après reperméabilisation post-vasectomie sont meilleurs que les taux de grossesse évolutive de $29 \%$ obtenus après MESA/ICSI. En outre, même la procédure de vaso-épididymostomie (VES), dont la réussite est moins bonne, donne encore des taux de grossesse spontanée de 31 à $56 \%$. De plus, le coût par accouchement après microchirurgie et après MESA/ICSI est largement en faveur du traitement chirurgical [13]. Avec le traitement chirurgical, de nombreuses complications et désavantages des techniques de reproduction in vitro peuvent être évités, comme le traitement hormonal de la partenaire, le recueil d'ovules et le transfert d'embryon transvaginaux, et le risque plus élevé pour la descendance d'anomalie chromosomique (sexuelle) après ICSI [16]. C'est pourquoi l'aspiration des spermatozoïdes épididymaires associée à l'injection intra-cytoplasmique d'un spermatozoïde devrait être réservée aux couples pour lesquels l'azoospermie obstructive ne relève pas de la reconstruction microchirurgicale.

\section{Vaso-épididymostomie}

Une procédure de vaso-vasostomie est indiquée si des spermatozoïdes sont trouvés dans la partie proximale du canal déférent lors de la chirurgie. Les spermatozoïdes peuvent être habituellement retrouvés dans un liquide séminal clair ou blanc ; un liquide crémeux à type de "pâte dentifrice" épaisse ne contient pas habituellement de spermatozoïdes et se voit souvent en cas de blocage épididymaire [1]. L'absence de liquide ou de spermatozoïdes est une indication à réaliser un examen de l'épididyme avec une aspiration microchirurgicale de spermatozoïdes épididymaires (MESA) et une vaso-épididymostomie. Les obstructions épididymaires apparaissent plus fréquemment après un intervalle de temps long entre la vasectomie et la reperméabilisation chirurgicale, et elles sont dues à un éclatement du canal épididymaire avec issue extracanalaire du liquide épididymaire et des spermatozoïdes entraînant secondairement une fibrose et une sténose de l'organe.

La vaso-épididymostomie (VES) est une procédure techniquement exigeante qui requiert une grande habileté en microchirurgie. La vaso-tubulostomie termino-latérale a été popularisée par Silber [23]. Les taux de reperméabilisation rapportés après une telle procédure vont de 64 à $78 \%$, et les taux de grossesse spontanée de 31 à $56 \%$ [18]. Le passage à travers une importante portion de l'épididyme est indispensable à la capacité fécondante des spermatozoïdes. Une anastomose entre le canal déférent et la tête de 
l'épididyme ne permet pas habituellement d'obtenir une grossesse spontanée. Les procédures de MESA/ICSI sont indiquées pour ces couples.

Récemment, Berger a introduit une modification de la technique d'invagination pour faciliter l'anastomose entre le canal épididymaire et la lumière du canal déférent, avec des résultats élevés de reperméabilisation initiale [3]. Le canal épididymaire dilaté est mis à nu par une petite incision de la tunique vaginale, et une légère pression est exercée sur l'épididyme par le pouce et l'index. Trois sutures doublepoint au nylon 10-zéro sont mises en place à travers la paroi antérieure du canal avant l'ouverture de la lumière et sont fixées de l'intérieur vers I 'extérieur de la muqueuse du canal déférent de façon triangulaire. Une tubulostomie est réalisée au milieu des trois sutures et le fluide séminal est examiné à la recherche de spermatozoïdes. L'anastomose est créée par cette méthode d'invagination en six sites pour l'alignement de la muqueuse, en utilisant seulement trois sutures double-point. La procédure a récemment été modifiée par Marmar avec l'utilisation de seulement deux sutures double-point 10-zéro [17]. D'autres auteurs ont aussi montré les avantages des techniques d'invagination sur un modèle animal [20] ; la technique d'invagination est plus facile à apprendre que la procédure latéro-terminale et est aussi efficace. Dans de courtes séries, le taux de reperméabilisation s'élève à $90 \%$ et cela dès 3 mois après la chirurgie [3]. Pour obtenir les meilleurs résultats, le chirurgien qui fait une reperméabilisation doit être capable de réaliser une vaso-épididymostomie en cas d'obstruction secondaire de l'épididyme, ce qui survient fréquemment chez les hommes ayant un long intervalle de temps entre la vasectomie et la reperméabilisation [5].

\section{CONCLUSION}

La réparation microchirurgicale de l'infertilité obstructive est un défi pour le médecin, mais de bons résultats sont tributaires de l'apprentissage et de l'expérience. La fécondité peut souvent être restaurée ou améliorée, évitant ainsi le recours aux techniques de reproduction artificielles [24]. Les procédures peuvent être combinées avec l'aspiration de spermatozoïdes et leur cryopréservation en vue d'une utilisation en ICSI en cas d'échec de la microchirurgie.

La comparaison de la microchirurgie et de la MESA/l CSI est largement en faveur de la microchirurgie tant en ce qui concerne les résultats que les coûts.

L'Urologue qui s'intéresse à l'Andrologie doit être encouragé à apprendre la microchirurgie comme constitutive de l'apprentissage chirurgical. Les meilleures conditions de réalisation de ces interventions sont les centres de médecine de la reproduction qui offrent une plus grande possibilité d'options différentes dans les cas particuliers.

\section{ABSTRACT}

Microsurgical vasectomy reversal : results and predictors of success

Gert R. DOHLE, Marij SMIT

Microsurgical vasectomy reversal is a challenge for the physician but successful treatment depends on the experience and skills of the surgeon. Fertility can often be restored, thus avoiding the need for artificial reproductive techniques. Also, the surgical procedures can be combined with sperm aspiration and cryopreservation, to be used for Intracytoplasmic sperm injection (ICSI) in cases of surgical failure. We describe the results of 217 vasovasostomy procedures, with special emphasis on recent technical refinements and prognostic indicators.

Between 1998 and 2002 we performed 217 vasovasostomy-procedures in an outpatient clinic setting. Refertilisation was successful in $76.5 \%$, spontaneous pregnancy occurred in $\mathbf{4 2} \%$ of the couples after a follow-up of at least 1 year. The main prognostic factors determining the outcome of the surgery was the interval between vasectomy and refertilisation and the age of the female partner: patency was $89 \%$ after an interval of less than 5 years and pregnancy occurred in $56 \%$ of these couples. After an interval of more than $\mathbf{1 0}$ years patency decreased to $75 \%$ and pregnancy results dropped to $24 \%$. Epididymal dysfunction with poor motility score and secondary epididymal obstruction appeared to be common after a long interval. Furthermore, in men with partners older than $\mathbf{3 5}$ years of age pregnancy was only $21 \%$, indicating limited ovarian reserve as an important factor in determining the final outcome.

In men with a long obstructive interval between vasectomy and reversal an obstruction of the epididymis can be found due to a blow-out of the epididymal tubule with subsequent leakage of semen in the organ and fibrosis. A vaso-epididymostomy procedure is needed to treat the obstruction. Recently, surgical refinements, such as the invagination technique, have been introduced for the vaso-epididymostomy procedure, showing promising first results. This simplified technique enables less experienced microsurgeons to perform this difficult operation successfully. The results of vasectomy reversal procedures can be improved substantially if the surgeon is able to perform a vaso-epididymostomy in cases of a secondary epididymal obstruction, occurring in about $25 \%$ of men with an interval of more than $\mathbf{1 0}$ years.

Key words : male infertility, microsurgery, vasectomy reversal, vasoepididymostomy 


\section{REFERENCES}

1. ANGER J.T., GOLDSTEIN M. : Intravasal "toothpaste" in men with obstructive azoospermia is derived from vasal epithelium, not sperm. J. Urol., 2004, $172:$ 634-636.

2. BELKER A.M., THOMAS A.J., FUCHS E.F., KONNAK J.W., SHARLIP I.D. : Results of 1469 microsurgical vasectomy reversals by the vasovasostomy group. J. Urol., 1991, 145 : 505511.

3. BERGER R.E. : Triangulation end-to side vasoepididymostomy. J. Urol., 1998, 159 : 1951-1953.

4. CARBONE D.J., SHAH A., THOMAS A.J., AGARWAL A. : Partial obstruction, not antisperm antibodies, causing infertility after vasovasostomy. J. Urol., 1998, $159: 827-830$.

5. CHAWLA A., O'BRIEN J., LISI M., ZINI A., JARVI K. : Should all urologists performing vasectomy reversals be able to perform vasoepididymostomies if required? J. Urol., 2004, 172 1048-1050.

6. DECK A.J., BERGER R.E. : Should vasectomy reversal be performed in men with older female partners ? J. Urol., 2000,163: 105-106.

7. ENGELMANN U.H., SCHRAMEK P., TOMAMICHEL G. DEINDL F., SENGE T. : Vasectomy reversal in central Europe: results of questionaire of urologists in Austria, Germany and Switzerland. J. Urol., 1990, $143:$ 64-67.

8. FOX M. : Failed vasectomy reversal : is a further attempt worthwhile using microsurgery ? Eur. Urol., 1997, $31: 436-440$.

9. GOLDSTEIN M. : Microsurgical vasovasostomy : Surgical approach, decision-making, and multilayer microdot technique. In : Goldstein M. ed. Surgery of male infertility. London, The Saunders Company, 1995, vol. $1: 46-60$.

10. HEIDENREICH A., ALTMANN P., ENGELMANN U.H. : Microsurgical vasovasostomy versus microsurgical epididymal sperm aspiration/testicular extraction of sperm combined with intracytoplasmic sperm injection. Eur. Urol., 2000, 37 : 609614.

11. HENDRY W.F. : Azoospermia and surgery for testicular obstruction. In : Hargreave T.B. ed. Male infertility. London, Springer Verlag, 1994 : 337-363.

12. HERNANDEZ J., SABANEGH E.S. : Repeat vasectomy reversal after initial failure : overall results and predictors for success. J. Urol., 1999, $161:$ 1153-1153.

13. KOLETTIS P.N., THOMAS A.J. : Vasoepididymostomy for vasectomy reversal : a critical assessment in the era of intracytoplasmic sperm injection. J. Urol., 1997, 158 : 467-470.

14. KOLETTIS P.N., SABANEGH E.S., D'AMICO A.M., BOX L., SEBESTA.M., BURNS J.R.. : Outcomes for vasectomy reversal performed after obstructive intervals of at least 10 years. Urology, 2002, $60: 885-888$.

15. KOLETTIS P.N., SABANEGH E.S., NALESNIK J.G., D'AMICO A.M., BOX L.C., BURNS J.R. : Pregnancy outcomes after vasectomy reversal for female partners 35 years old or older. $\mathrm{J}$. Urol., 2003, $169: 2250-2252$.

16. KURINCZUK J.J : Safety issues in assisted reproduction technology. From theory to reality-just what are the data telling us about ICSI offspring health and future fertility and should we be concerned ? Hum. Reprod., 2003, 18 : 925-931

17. MARMAR J.L. : Modified vasoepididymostomy with simultaneous double needle placement, tubulotomy and tubular invagination. J. Urol., 2000,163: 483-486.

18. MATTHEWS G.J., SCHLEGEL P.N., GOLDSTEIN M. : Patency following microsurgical vasoepididymostomy and vasovasostomy : temporal considerations. J. Urol., 1995, 154 : 2070-2073.
19. MEINERTZ H., LINNET L., FOGH-ANDERSEN P., HJORT T. : Antisperm antibodies and fertility after vasovasostomy : a follow-up study of 216 men. Fert. Steril., 1990, $54: 315-321$.

20. POPKEN G., WETTERAUER U., SCHULTZE-SEEMANN W. : Microsurgical epididymovasostomy in the rat : comparison of end-to-side and two invagination techniques. Urol. Res., 1999, 27 : 214-218.

21. ROSENWAKS Z., DAVIS O., DAMARIO M. : The role of maternal age in assisted reproduction. Hum. Reprod., 1995, 10 : 165-173.

22. SHARMA R., AGARWAL A. : Role of reactive oxygen species in male infertility. Urology, 1996, $48: 835-850$.

23. SILBER S.J. : Results of microsurgical vasoepididymostomy : role of the epididymis in sperm maturation. Hum. Reprod., $1989,4: 298-303$.

24. SILBER S.J., GROTJAN H.E. : Microscopic vasectomy reversal 30 years later : a summary of 4010 cases by the same surgeon. J. Androl., 2004, 25 : 845-859.

Manuscrit reçu : janvier 2005 ; accepté février 2005. 